\title{
1 High frequency rTMS modulates functional connectivity nodes and 2 boundaries differently in the human brain
}

3 Short title: HF-rTMS changes brain connectivity nodes and boundaries

4

5

6

\# equal contribution

\section{*Corresponding author:}

Dr. Roberto Goya-Maldonado Laboratory of Systems Neuroscience and Imaging in Psychiatry (SNIP-Lab) Department of Psychiatry and Psychotherapy University Medical Center Göttingen (UMG) Von-Siebold Str. 5, 37075 Göttingen e-mail: roberto.goya@med.uni-goettingen.de

$\begin{array}{lllll}\text { Abstract words: } & 177 & \text { Figures: } & 6 & \text { Suppl Figures: } 3 \\ \text { Text words (incl M\&M): } & 6067 & \text { Tables: } & 0 & \text { Suppl Tables: } 2 \\ \text { References: } & 66 & & & \end{array}$


35

36

37

\section{Abstract}

Repetitive transcranial magnetic stimulation (rTMS) has gained considerable importance in the treatment of disorders, e.g. depression. However, it is not yet understood how rTMS alters brain's functional connectivity. Here we report the changes captured by resting state functional magnetic resonance imaging (rsfMRI) within the first hour after $10 \mathrm{~Hz}$ rTMS in (1) nodes, where the strongest functional connectivity of regions is seen, and (2) boundaries, where functional transitions between regions occur. We use support vector machines (SVM), a widely used machine learning algorithm that has been proven to be robust and effective, for the classification and characterization of time intervals of major changes in node and boundary maps, while respecting the variability between subjects. Particularly in the posterior cingulate cortex and precuneus, our results reveal that the changes in connectivity at the boundaries are slower and more complex than in those observed in the nodes, but of similar magnitude according to accuracy confidence intervals. As the network boundaries are under studied in comparison to nodes in connectomics research, our results highlight their contribution for functional adjustments to rTMS.

(1)

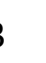



5

6

57

8

59

60

61




\section{Introduction}

Repetitive transcranial magnetic stimulation (rTMS) has become a popular method for the non-invasive modulation of brain function (George et al., 1999). Recent neuroimaging studies have shown that functional changes induced by rTMS in a localized cortical region lead to selective and distinct modulation of activity and functional connectivity both within and between large-scale brain networks (Cocchi et al., 2015; Eldaief et al., 2011; Sale et al., 2015; Singh et al., 2020, 2019; Valchev et al., 2015; Watanabe et al., 2014). The mechanisms by which rTMS induces network modulations are still not well understood. Mapping whole-brain effects caused by local neural perturbations, including by rTMS, is a growing field of research. Well-established methods now allow for the assessment of connectome-level functional adjustments to high frequency rTMS in both node and boundary maps in sequential time intervals (Cohen et al., 2008; Wig et al., 2014).

Functional magnetic resonance imaging ( $\mathrm{fMRI}$ ) data obtained while participants are not engaged in any specific task is called resting state fMRI (rsfMRI). RsfMRI has been instrumental for advancing our understanding of the brain's macroscopic functional network architecture (Power et al., 2011; Smith et al., 2013; Yeo et al., 2011) as well as which regions might be most functionally altered in psychiatric disorders (Goya-Maldonado et al., 2016; Wei et al., 2018). However, fMRI data typically consists of functional time-courses in thousands of voxels, which on the one hand allows for accurate inference of correlations or "functional connectivities" between regions, but on the other hand has high dimensionality. Different approaches have been proposed to reduce data dimensionality of the data and to identify the most relevant patterns of spatiotemporal organization in $\mathrm{fMRI}$ data. This is the case for whole-brain functional regions that will be represented in our study as nodes (Stanley et al., 2013) and boundaries (Cohen et al., 2008; Gordon et al., 2016; Tourville et al., 2019). Nodes are defined as the greatest strength of local or global connectivity, also known as concepts of modularity and integration respectively, which enabled many insights into dimensional organization of the healthy and diseased brain (van den Heuvel and Sporns, 2019). Boundaries are the counterpart of the nodes, identified where the connectivity strength is the lowest or absent, usually in the transition between neighboring functional regions. In contrast to the investigation of boundaries, the scientific community has given disproportionate attention to 
the nodes of functional networks. In node clustering approaches, spatiotemporal elements (i.e., voxels) may be grouped on the basis of the similarity versus dissimilarity of their functional connectivity (Stanley et al., 2013). An example of a node clustering approach is independent component analysis (ICA), a brain mapping method that efficiently segregates functional components based on their corresponding spatiotemporal distribution. ICA has been widely applied to the identification of large-scale brain networks (Agosta et al., 2012; Peraza et al., 2014; Singh et al., 2020, 2019).

Group-based parcellation schemes use fMRI data from multiple individuals to map large-scale functional brain networks, that is, collections of widespread regions showing functional connectivity (Eickhoff et al., 2018; Glasser et al., 2016; Tourville et al., 2019). While groupbased parcellation captures major features of functional brain organization that are evident across individuals, such approaches may obscure certain person-specific features of brain organization. In contrast, subject-specific parcellation methods have been shown to effectively map aspects of functional organization that differ for particular individuals (e.g. Kong et al., 2019; Saxe et al., 2006). Several recent studies have demonstrated that extensive rsfMRI data collected across multiple sessions from the same individual can be used to delineate high-quality cortical parcellations at the individual level (Gordon et al., 2017; Laumann et al., 2015; Tavor et al., 2016). Subject-specific parcellation may enable increasingly precise planning and delivery of rTMS interventions. Toward this goal, an important consideration is $\mathrm{fMRI}$ signal to noise ratio (SNR), small value of which may obscure important effect of rTMS. A number of FMRI preprocessing steps can be helpful for improving SNR, including magnetic field inhomogeneities-based unwarping, head-motion regression, spike scrubbing, band-pass filtering and Gaussian kernel spatial smoothing. For personalized approaches, as previously employed in our research group (Singh et al., 2019), ICA can additionally improve SNR by separating signal from noise components.

Machine learning includes the development of algorithms that can model spatially complex and often subtle patterns, and has been applied to neuroimaging and the mapping of brain stimulation effects in individual subjects. Machine learning may thus be useful for individuallevel predictions that could ultimately be used in clinical contexts (Cao et al., 2020; Cash et al., 2019; Drysdale et al., 2017; Redlich et al., 2016). The support vector machine (SVM) is a machine learning algorithm that constructs hyperplanes in multidimensional space to 
optimally separate data classes (Evgeniou and Pontil, 2001). The resulting model can then be used to classify the data class of new data points. Taken together, in the current study SVM was used to identify the strongest rTMS-related changes in brain functional connectivity patterns in both nodes and boundary maps. In the domain of "big" datasets other highly nonlinear algorithms often outperform SVM (Liu et al., 2017). Alternatively, SVM often yields better classification in applications with smaller datasets, that is, datasets in which the number of features exceeds the number of training data samples (Chaplot et al., 2006). The classification task can be simplified by using unbiased feature selection approaches. Features selection involves the identification of the most useful data features in the training dataset (Miao and Niu, 2016). These features are then solely used for classification. Features selection has been shown to improve accuracy while also increasing the interpretability of identified multivariate models.

Recent neuroimaging and predictive modeling findings suggest that a locally generated brain stimulation-induced perturbation in neural activity is gradually integrated by selective alterations of within- and between-network dynamics (Cocchi et al., 2015; Gollo et al., 2017). In addition, animal studies have shown that $10 \mathrm{~Hz}$ rTMS creates a transient cortical functional state that is characterized by increased excitability and increased response variability (Kozyrev et al., 2018, 2014). The latter finding implicates a reduction of specificity (decorrelation) of the functionally distinct regions and the widening of boundaries between them. In the current project, we endeavored to understand rTMS modulation of whole-brain connectivity patterns by employing complementary approaches including assessment of both nodes and boundaries of functional brain networks. Our approach allowed for the data-driven identification of the most substantial functional changes across time. Analyzed data include timely precise rsfMRI measurements at baseline (RO) as well as following $10 \mathrm{~Hz}$ rTMS in the left dorsolateral prefrontal cortex (DLPFC) in continuous time intervals occurring 10-15 $\mathrm{min}, 27-32 \mathrm{~min}$, and 45-50 min after stimulation (intervals named R1, R2, R3). We used individual-level comparisons to create sham-corrected maps for nodes and boundaries. This approach enhanced the sensitivity of detecting individual-level rTMS-induced variations in functional connectivity. Given the multiple measurements of resting state functional connectivity patterns from R0 to R3 time intervals, we were able to sequentially map spatiotemporal changes related to stimulation. Based on the prior studies described above, we hypothesized 
that $10 \mathrm{~Hz}$ rTMS would affect functional connectivity in the nodes and in the boundaries of distant regions that interact with the DLPFC.

\section{Materials and Methods}

\section{Participants and study design}

23 participants were recruited from a university environment to participate in a double-blind, sham-controlled, cross-over design study that investigated the neural effects of $10 \mathrm{~Hz}$ rTMS using rsfMRI. Details on the design of the study have been reported in detail elsewhere (Singh et al., 2019). Participants were screened with a self-report clinical questionnaire and the Symptom Checklist 90 Revised (SCL-90-R) to ensure that they had no current or previous history of neurological or psychiatric disorders. Additional exclusion criteria included recreational drug use in the past month, current or history of substance abuse or addiction, any contraindications to MRI or TMS (e.g. epilepsy), pregnancy, history of traumatic brain injury, current use of anticonvulsant drugs, participation in any TMS or ECT study in the past 8 weeks, and unwillingness to consent or to be informed of incidental findings. Informed consent was obtained from all subjects before their inclusion in the study. The study protocol was approved by the Ethics Committee of the University Medical Center Göttingen.

For each participant, experiments were conducted over the course of 3 days with approximately one week in between each day. As described in Singh et al. (2019), on day 1 a structural T1-weighted volume and rsfMRI were acquired for the identification of a subjectspecific DLPFC site for rTMS stimulation. This target was then used for real and sham stimulation protocols on day 2 and day 3 . At the beginning of day 2 and day 3 , an rsfMRI scan (RO) was obtained pre-rTMS. Next the resting motor threshold (RMT) for each subject and session was determined, which was then used to set the stimulation intensity (i.e., $110 \%$ of the RMT). Thereafter, a $10 \mathrm{~Hz}$ rTMS clinical protocol of 3000 pulses was delivered over 37.5 min. This procedure was additionally controlled by sham rTMS in a double-blind counterbalanced crossover design. RTMS was precisely delivered to each subject at the preselected DLFPC target, guided by online neuronavigation. Three additional rsfMRI scans (R1, $\mathrm{R} 2$, and R3) were obtained post- rTMS at 10-15 $\mathrm{min}, 27-32 \mathrm{~min}$, and 45-50 $\mathrm{min}$ after the end 
of stimulation. These acquisitions allowed for the assessment of functional connectivity changes induced by rTMS.

\section{Neuroimaging data acquisition and preprocessing.}

Structural T1-weighted scans with 1-mm isotropic resolution and functional data were obtained using a 32-channel head coil and 3T MR scanner (Magnetom TRIO, Siemens Healthcare, Erlangen, Germany). For rsfMRI, 125 volumes were acquired in approximately 5.5 minutes using a gradient EPI sequence with the following parameters: TR of $2.5 \mathrm{~s}$, TE of $33 \mathrm{~ms}$, 60 slices with a multiband factor of 3 , FOV of $210 \mathrm{~mm} \times 210 \mathrm{~mm}, 2 \times 2 \times 2 \mathrm{~mm}$, with $10 \%$ gap between slices and anterior to posterior phase encoding.

RsfMRI preprocessing was conducted in Data Processing Assistant for Resting-State fMRI software (DPARSF V4.4, (Chao-Gan and Yu-Feng, 2010)). Initial steps of preprocessing included the slice timing and head motion correction (Satterthwaite et al., 2013; Yan et al., 2013). Afterwards corrected images were analyzed using the SPM12 gradient echo field map unwarping tool (Togo et al., 2017). White matter, CSF, and global signal were then regressed out to additionally reduce nuisance effects (Power et al., 2017). Corresponding T1-weighted images were adjusted to the standard Montreal Neurological Institute (MNI) template and then used for spatial normalization of rsfMRI with a resampled voxel size of $3 \times 3 \times 3 \mathrm{~mm}^{3}$. The preprocessed images were then spatially smoothed with a $4 \times 4 \times 4 \mathrm{~mm}$ full width at half maximum (FWHM) Gaussian kernel. Data were then detrended and band-pass $(0.01-0.1 \mathrm{~Hz})$ filtered to remove bias from low-frequency drift and high frequency noise.

\section{Parcellation of individual-level resting state functional correlations}

Individualized cortical parcellations were obtained using two methods: (1) the "Snowballing" algorithm provided a connectivity peak density map (Wig et al., 2014) and (2) Boundary Mapping generated an average spatial gradient map (Cohen et al., 2008; Wig et al., 2014). Both methods use whole-brain resting state functional connectivity (RSFC) to create individualized node and boundary maps. Briefly, the Snowballing algorithm uses seed-based RSFC to identify locations that are correlated (the "neighbors") with a starting seed region of interest (ROI) location. These neighbors are then used as the new seed regions and the procedure is repeated iteratively. Each iteration of identified neighbors in this procedure is 
referred as a "zone". The neighbors correlated with a given seed region are spatially clustered with their distinct local maxima. Averaged peak voxel locations over multiple zones results in a peak density map that represents the number of times a voxel was identified as a peak across all ROI correlation zones from starting seed locations. As in (Wig et al., 2014), nodes were identified as the peak distribution from three zones of Snowballing . These peak distribution maps have been shown to be invariant to the locations of starting seeds as well as the seed sizes. We used 278 initiation points that corresponded to 278 ROls (Power et al., 2011) and a $\mathrm{ROI}$ radius of $4.5 \mathrm{~mm}$. The correlation maps were thresholded at $r>0.3$.

In contrast to the definition of nodes, RSFC-Boundary Mapping identifies locations where the patterns of RSFC correlations exhibit abrupt transitions, and therefore provides estimates of putative boundaries between functional regions (Cohen et al., 2008). In contrast to the initially proposed technique that flattens a given subject's cortex into a 2D surface (Wig et al., 2014), we performed all computations directly on the subject's full-volume 3D Cartesian grid. This allowed us to simultaneously overlay, for each subject, the 3D Snowballing peak density images with the RSFC-Boundary Mapping output (Figure 1). Nevertheless, similarly to the original report, we also found that gradients resulting from RSFC-Boundary Mapping (Figure 1, in green) typically surrounded the nodes of high peak count identified by the Snowballing algorithm (Figure 1, in red) Aside from this difference, we followed the RSFC-Boundary Mapping method as previously described (Wig et al., 2014). The full-resolution preprocessed fMRI data were first resampled to a coarser grid, resulting in 19,973 voxels $\left(4.5 \times 4.5 \times 4.5 \mathrm{~mm}^{3}\right.$ in size). The whole-brain RSFC map was then computed for each voxel by correlating the time series of the given voxel with every other voxel. Then, the similarity between each voxel's temporal correlation map and every other voxel's temporal correlation map was computed as the spatial correlation between them. That resulted in $19,973 \times 19,973$ symmetrical spatial correlation matrices. Every row of this matrix corresponds to a reference voxel and can be displayed in the volume of the brain where the value in every voxel is the similarity between the temporal correlation map at that position and the reference voxel. Every row was then mapped to the brain mask back, representing voxel's similarity map. Next the spatial gradient was computed and then averaged across all similarity maps. The average of those spatial gradient maps represents the probability with which each location is identified as a point of rapid change in the RSFC maps between two neighboring areas. Both node and boundary maps were further smoothed with a $4 \times 4 \times 4 \mathrm{~mm}$ FWHM Gaussian filter. 


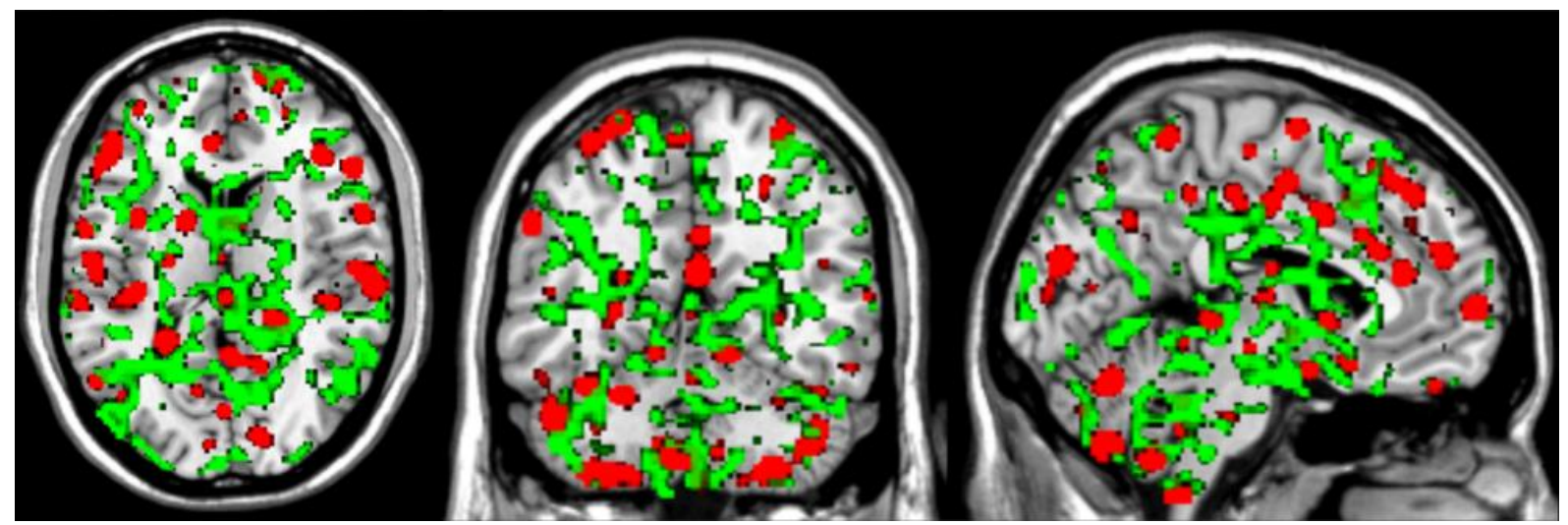

Figure 1: An example of individual-level node and boundary maps. It is noteworthy that calculated RSFC-Snowballing nodes (red areas, thresholded $r=0,3$ ) are often surrounded by transitional areas represented by RSFC-Boundary mapping boundaries (green areas thresholded $r=0,08)$, revealing the complementary nature of both parcellation methods.

\section{Defining the regions of interest}

256

257

258

259

260

261

262

263

264

265

266

267

268

269

Machine learning models can be directly applied to both node and boundary maps. However, due to a large number of features $\left(10^{4}-10^{5}\right.$ voxels) compared to the number of subjects in this study $(n=23)$, that would lead to a substantial overfitting. Subsequently, overfitted classification models suffer from unsatisfactory interpretability and accuracy (Khosla et al., 2019; Kohoutová et al., 2020). In order to select a smaller number of physiologically plausible voxels located in node and boundary maps, we employed a novel data-driven feature selection approach based on the resting state networks (RSNs). RSNs were accessed in the same group of subjects but from an independent dataset (Day 1) from the classification dataset (Day 2 and 3). The RSNs were identified by group ICA (Khosla et al., 2019; Smith et al., 2013) using MELODIC tool of FSL 5.0.7. We temporally concatenated the fMRI data of 23 subjects recorded on Day 1. Based on visual inspection and the power spectrum of the MELODIC output, we selected the nine best-fitting spatiotemporal independent components (IC). While the node regions were assumed to lie close to maxima of the selected ICs, the boundary areas were rather identified at the intersections of the ICs. 


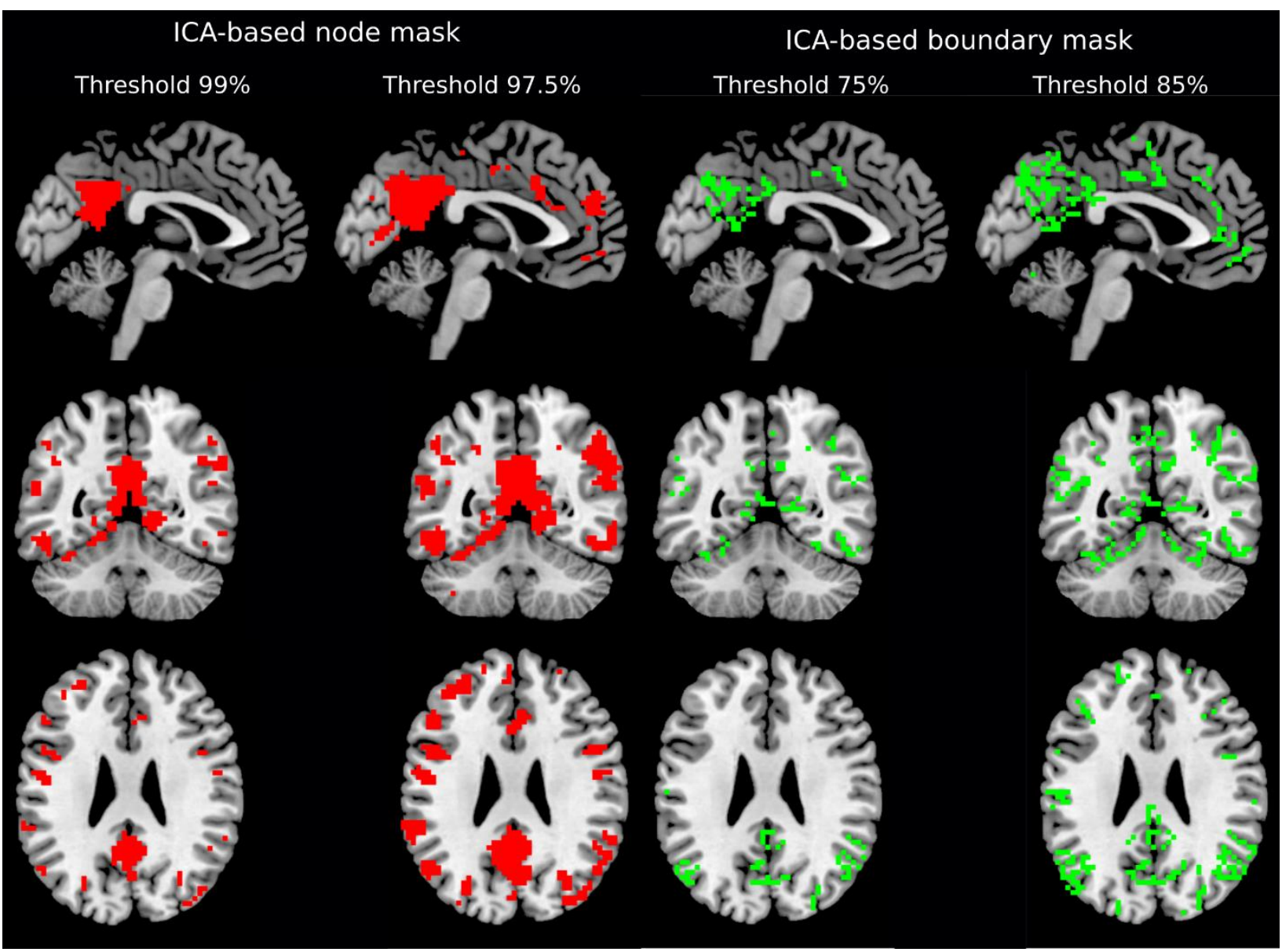

272 Figure 2: ICA-based binary masks of node (left) and boundary masks (right) from a dataset not used in machine learning classification. ICs node regions grow in size when one increases the

274 threshold value. At the moment two or more ICs meet in one particular voxel, that voxel is 275 identified as the boundary. Changing the threshold value allows for the optimization of the number of voxels corresponding to both masks.

As the different ICs showed different strengths (distributions of values across the brain) and we intended to have multiple networks represented rather than a single "winner", the node areas were defined by overlaying of all ICs. The percentage of voxels belonging to every IC was controlled by the threshold; voxels with lower strength were discarded. Next, the components were merged together resulting in an array of nodal voxels. The value of the peak density

283 (Snowballing) map of each subject/time interval was then extracted for each of those voxels.

284 This process was designed to select the most important features that would then be modeled 
using the classification algorithm. An example set of node masks for a specific subject is presented in Figure 2 (left; thresholded at $97.5 \%$ and $99 \%$ of discarded voxels).

In case of the boundary mask, we have developed an algorithm closely related to watershed transform (Kolade et al., 2014). Underlying idea of watershed transform is finding an optimal position for dams to be built, where the water coming from different basins, according to the surface shape, would meet. In our case the surface is represented by all ICs and the basins are the strongest voxels according to ICs. Starting from the maximum of each component (100\% threshold), and then reducing the threshold in $1 \%$ steps, the ICs increase in size. A voxel is identified as a boundary when two or more ICs intersect each other (i.e., the same voxel is included in multiple ICs). Single voxels, i.e. not surrounded by the voxels from the same IC, were not treated as boundaries to avoid spurious findings. The number of included voxels were dependent on the percentage level step that was used to descend from the maximum. An example set of boundary masks for a specific subject is presented in the Figure 2 (right; threshold values of $75 \%$ and $85 \%$ ). The resulting array of voxels was then used with the gradient maps for machine learning classification.

\section{$\underline{\text { SVM classification }}$}

The capacity of SVM to predict outcomes of the rTMS intervention was first assessed using leave-one-group-out stratified cross-validation (CV), where the group was defined as a pair of stimulation conditions (time intervals) of the same subject. Our main analysis employed SVM for binary classification of the six sham-subtracted pairs of conditions: R0 vs R1; R0 vs R2; R0 vs R3; R1 vs R2; R1 vs R3 and R2 vs R3. SVM classification was performed using a linear kernel and the default scaling factor of 1 . Sex and age were not regressed out because in every comparison both groups were represented by the same subjects, and thus automatically balanced.

The number of features (voxels) used as an input for SVM varied by changing the threshold value of the extracted ICA-based node and gradient masks. SVM assigns weight to every voxel, which can be interpreted as an importance of the voxel to separate conditions. The SVM was trained for every pair of conditions starting with a mask threshold corresponding to 10,000 voxels. By changing the threshold for both ICA-based node and gradient masks, we removed the "weakest" voxels and trained the SVM again from scratch. This procedure was repeated 
until the total number of voxels surviving thresholding reached zero. This recursive process allowed for the assessment of a model accuracy curve that was defined by the percentage of included voxels. The global maximum of this model accuracy curve represents the most informative set of voxels for separating the particular pair of conditions. The same algorithm was applied to both the peak density and the gradient maps across voxel thresholds. This procedure is schematized in Figure 3.

$\mathrm{CV}$ is well known as an effective method in machine learning for testing generalizability of a trained model. Model performance was further tested using leave-two and leave-three-out stratified CV ( 7 fold and 11 fold CV) in which 2 or 3 subjects were withheld from training and assigned to the test sample. Our goal with this approach was to investigate the effect of the amount of data provided to a classifier, and thereby assess the impact of CV strategy. By providing more training data, as in leave-one-out $\mathrm{CV}$, the model has more generalizable performance compared to leave-two-out and leave-three-out CV (Wottschel et al., 2019). In addition, we calculated across all conditions the receiver operating characteristic (ROC) area under curve (AUC). This was used as an aggregate measure of performance across the whole range of classification thresholds (see Supplementary Table 1). We performed a bootstrap test to assess the stability of the most informative voxels (i.e., the stability of each voxel's contribution to classification contribution across conditions). That is, first we randomly resampled the training samples with replacement. Stability of the voxel was then evaluated by using $z$ and $p$ values of the voxel's weight according to the trained SVM. Voxels with $p<0.05$ were considered stable, while voxels with $p>0.05$ were discarded. The computations were completed in Python using custom-written scripts that used functions from the Nilearn v0.7.0 (https://nilearn.github.io/) and Sklearn v0.23.2 (https://scikit-learn.org/stable/) libraries. 


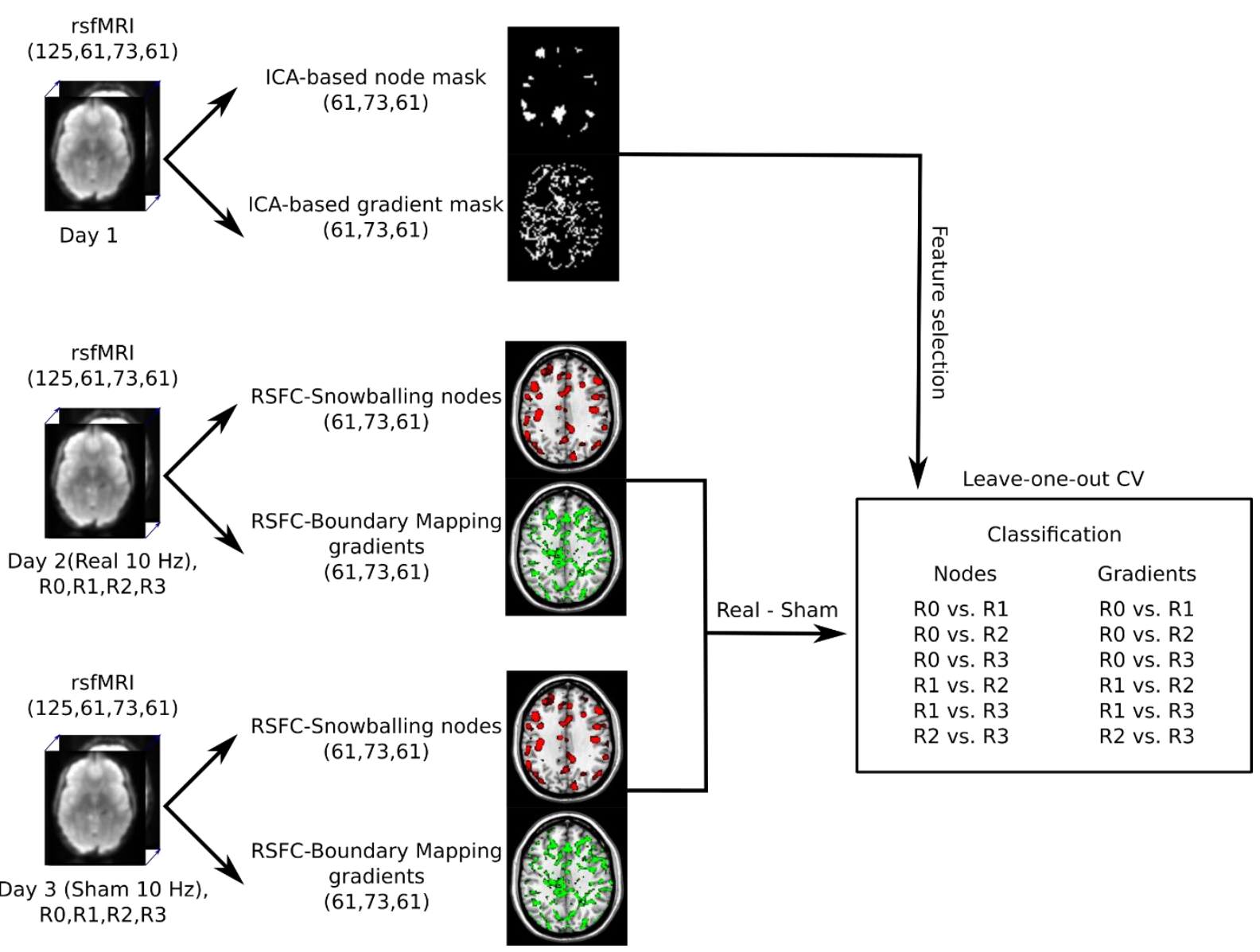

Figure 3: Schematic diagram of the analysis pipeline. RsfMRI data from 4 sessions (R0, R1, R2, R3). Both rsfMRI from Day 2 (real rTMS) and Day 3 (sham rTMS) were used to compute RSFCSnowballing density nodes and RSFC-Boundary Mapping gradients. Next sham stimulation maps were subtracted from the corresponding real stimulation maps. The extracted ICA-based masks derived from the Day 1 measurements were then applied to the corresponding node and gradient maps for feature selection. Finally, the remaining voxels were used for machine learning classification.

Effect of head motion on the separation of conditions.

To exclude the possibility that condition-related differences are caused by head motion (Van Dijk et al., 2012), we performed pairwise classification of mean frame-to-frame head displacement across real, sham, and real-sham conditions (R0, R1, R2, R3) . The resulting machine learning performance accuracies are presented in Supplementary Table 2. As the performance of algorithms based on head motion are close to chance, this analysis confirmed no influence of head motion on the results of classification based on RSFC peaks and boundary maps. 
Results

\section{Analysis based on RSFC peaks density maps}

358

359

360

361

As our intention was to discriminate the sessions in which connectivity was most strongly modulated by $10 \mathrm{~Hz}$ rTMS, we first identified which comparisons of the Snowballing node maps revealed the highest discrimination as measured by SVM accuracy. The threshold value for the ICA-based node mask, representing the percentage of removed voxels, varied between $99.9 \%$ (154 voxels) to $94.5 \%(10,799$ voxels) with steps of $0.1 \%$. The highest accuracy of $74.2 \%$ was achieved for R1 vs. R2 with a threshold value of $99.7 \%$, yielding 764 voxels used in SVMbased classification (Figure 4a). Results indicate that a sparser ICA-based node mask did not contain enough information to discriminate R1 from R2. Furthermore, increasing the number of voxels resulted in decreasing accuracy, which may be due to model overfitting (i.e., inclusion of uninformative voxels). We additionally compared the performance of models that used different CV routines (Figure 4b). All three CV schemes yielded similar accuracies across thresholds ranging from $99.9 \%$ to $98.7 \%$. Lower thresholds for leave-three-out CV resulted in higher accuracy compared to leave-one-out and leave-two-out CV. This may indicate overfitting due to the large number of voxels included in classification. Results for all other comparisons across CV strategies are included in Supplementary Figure 1. Finally, AUC for R1 vs. R2 with 764 voxels used with SVM yielded $0.76 \pm 0.02$.

For feature-level assessment we used a bootstrapping-based resampling approach. This resulted in the quantification of feature stability. For the highest SVM classification performance (i.e., R1 vs. R2 with a threshold value of 99.6\%), 634 of 764 voxels were found stable $(p<0.05)$ with both positive and negative weights located in posterior cingulate cortex, angular gyrus, anterior insula, and visual cortex (Figure 6). 

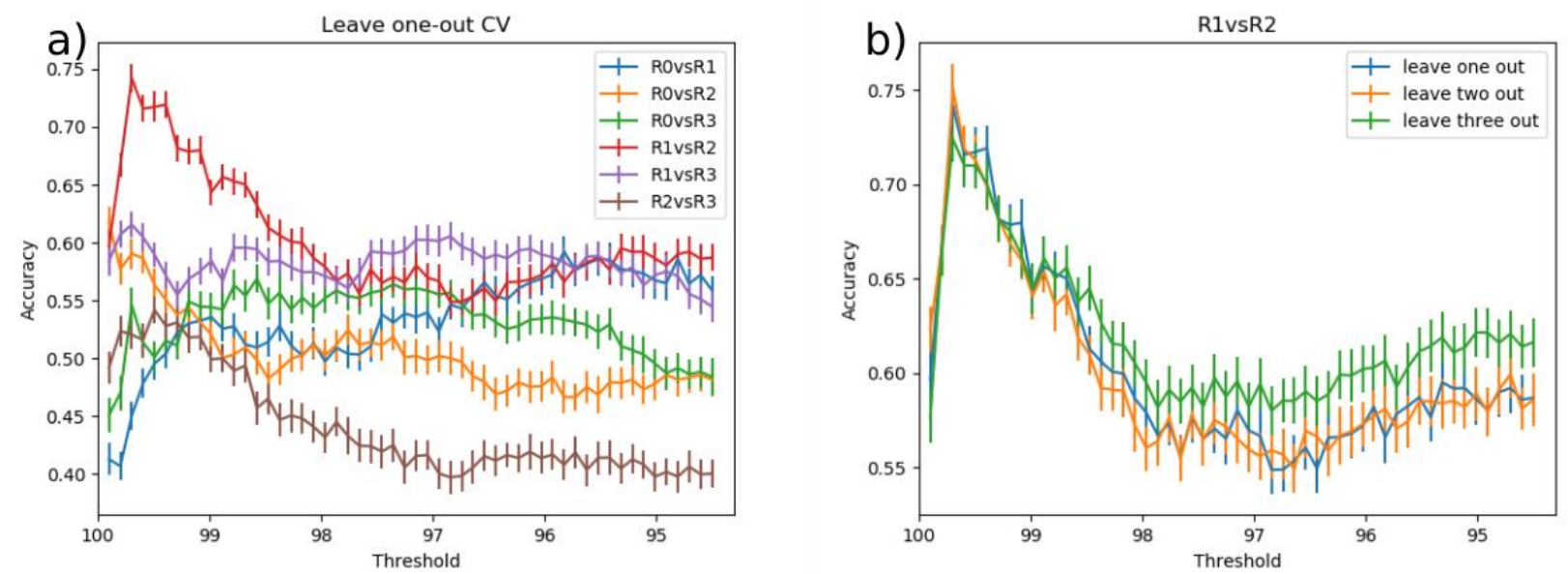

Figure 4: Condition classification accuracy of SVM using peak density map features. (a) Performance of models for all pairs of conditions across all threshold levels. The error bar indicates 95\% confidence intervals over 1000 bootstraps. The highest performance was achieved for R1 vs. R2 for the threshold value of $99.7 \%$. This threshold value corresponded to 764 voxels in the mask. Note that by lowering the threshold, i.e., increasing the number of voxels, classification accuracy decreases. (b) To test the stability of classification results, classification was also performed for leave-one-, -two- and -three-out CV routines. For the threshold value of $99.7 \%$, leave-one-out and leave-two-out CV yielded higher classification accuracy compared to leave-three-out CV strategy.

\section{Analysis based on RSFC Boundary maps}

For extracted gradients from RSFC Boundary Mapping, the threshold for ICA-based gradient masks varied from $60 \%$ (113 voxels) to $94 \%$ (10861 voxels) with steps of $1 \%$. The highest SVM accuracy of $74.5 \pm 1.1 \%$ was achieved for R1 vs. R3 (Figure 5A) for the threshold value of $63 \%$ (174 voxels), and yielded 0.80 \pm 0.02 AUC (Supplementary Table 1). Using leave-one-out CV resulted in the performance improvement from $72.3 \%$ to $74.5 \%$ compared to leave-two-out CV for the ICA-based gradient mask threshold of $63 \%$. Consistent with the analysis on Snowballing nodes described above, increasing the number of voxels extracted from the ICAbased gradient mask caused a drop in the overall accuracy of the SVM. This result suggests an increasing number of irrelevant or redundant voxels. The learning curve of R1 vs. R3 across CV approaches (Figure 5B) indicates the highest masking threshold at which leave-one-out CV strategy yields the highest (or at least equal accuracy compared to leave-two and leave-three- 
402

403

404

405

406

407

408

409

410

411

412

413

414

415

416

out CV) was 76\%. This masking threshold corresponded to 1194 voxels. For higher threshold values, leave-two-out CV exhibited slightly higher accuracy than the other approaches. Leaveone-out CV resulted in performance improvement from $72.3 \%$ to $74.5 \%$ for the ICA-based gradient mask threshold of $63 \%$. Out of 174 voxels, 165 showed feature stability in bootstrapping procedure as indicated by $p<0.05$. These voxels were predominantly located in the ventral posterior cingulate cortex, angular gyrus, and fusiform gyrus (Figure 6).

The second highest SVM performance accuracy was $68.5 \pm 1.3 \%$ for R1 vs. R2 (Figure 5a). This performance was achieved using a threshold value of $67 \%$ and corresponded to 346 included voxels. Of the 346 voxels 329 survived the stability procedure via bootstrapping. The corresponding AUC yielded 0.75 \pm 0.02 (Supplementary Table 1). For threshold values from $60 \%$ to $86 \%$, both leave-one-out and leave-two-out CV strategies were associated with higher accuracy compared to leave-three-out CV (Figure 5C). The sign of feature weights in the majority of identified voxels was in the opposite direction to the R1 vs. R3 comparison. This sign change suggests fluctuations in the functional connectivity between time points R2 and R3 (see Discussion). 

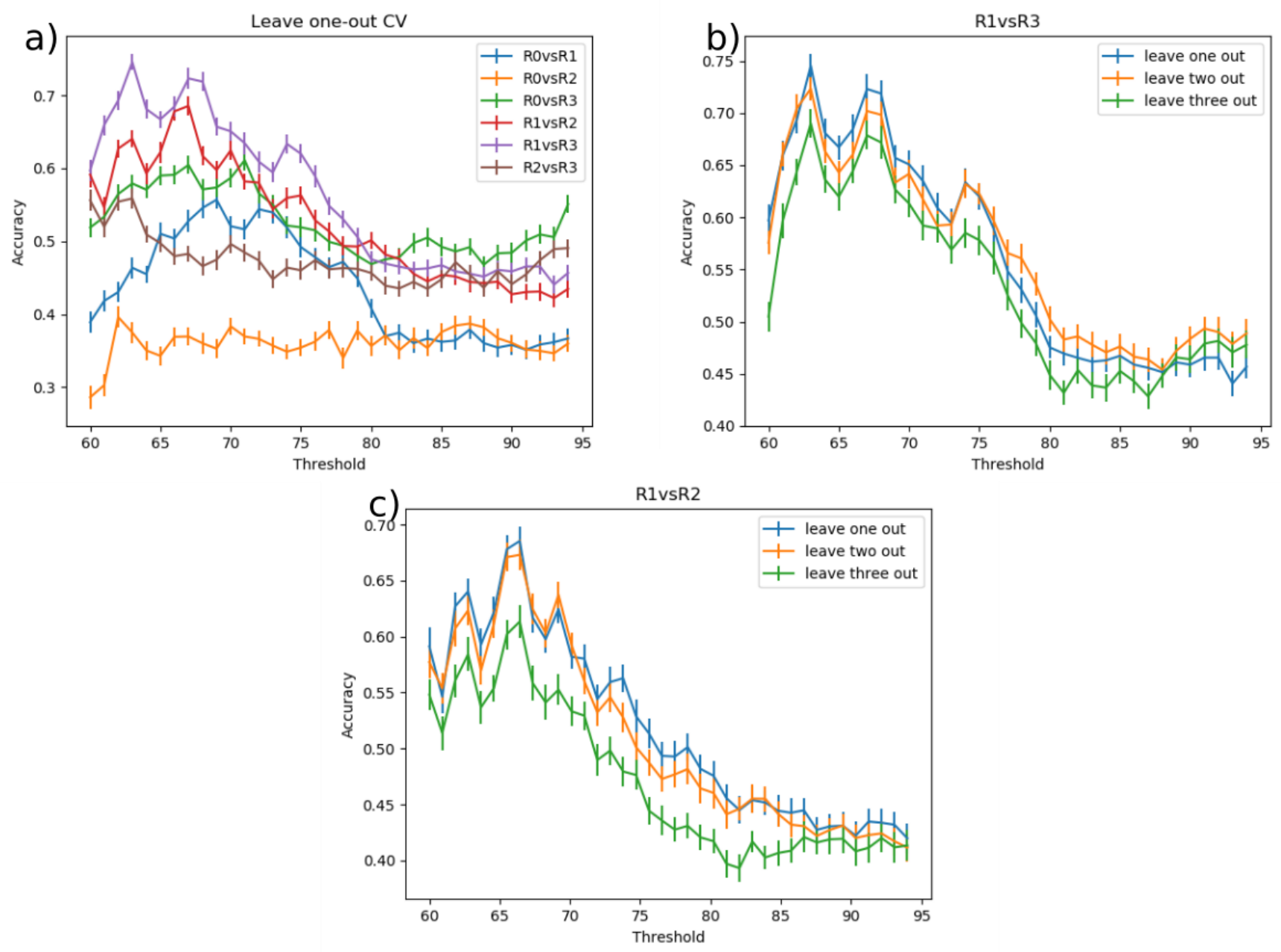

Figure 5:

Classification accuracy of SVM using RSFC boundary features (gradient maps) across condition. (a) Accuracy for all comparisons. The error bar indicates 95\% confidence intervals over 1000 bootstraps. The accuracy was the highest for the R1 vs. R3 comparison, reaching $76 \%$ at the threshold value of $63 \%$. This threshold value corresponded to 165 voxels in total. (b) To access the effect of data size and stability of classification results, we run leave-two- and leave-threeout CV routines. The accuracy for different CV routines suggests that there is a high number of informative voxels for threshold values smaller than $76 \%$. (c) Second highest performing comparison. R1 vs. R2 discriminative performance was $68.5 \%$. The stability analysis at the threshold value of $67 \%$ corresponded to 346 extracted voxels. 


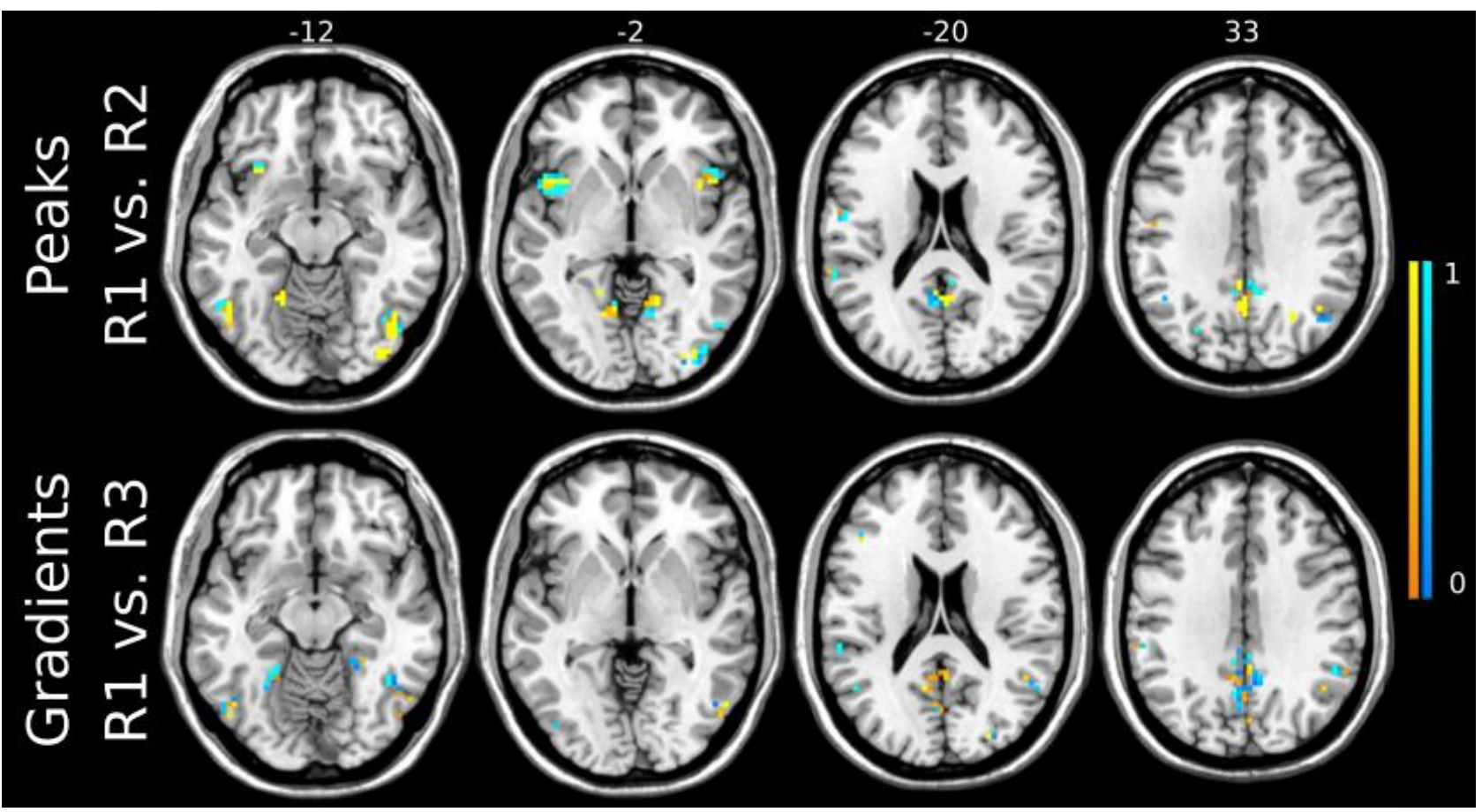

$430 \quad$ Figure 6: Voxels corresponding to the highest performance of the SVM using the Snowballing

431 maps for R1 vs. R2 comparison (top) and the Boundary-Mapping gradients for R1 vs. R3

432 comparison (bottom). The sign and strength of modulation is color-coded by warm colormap

433 (connectivity increase) and cold colormap (connectivity reduction). The numbers refer to axial

434 plane z-coordinates in MNI space.

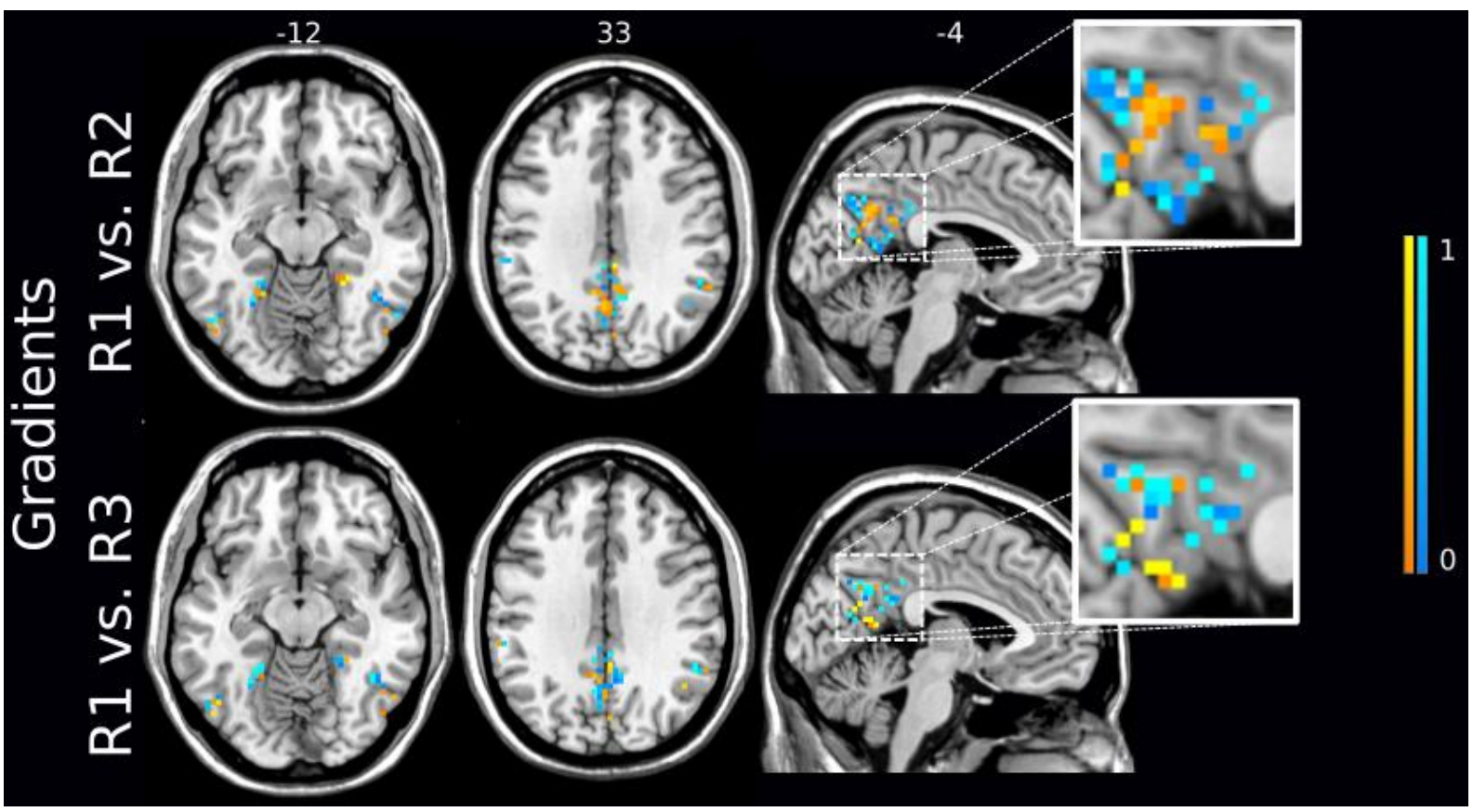


Figure 7: Two highest classification accuracies based on the Boundary-Mapping gradients were achieved for comparisons R1 vs. R2 (top) and R1 vs. R3 (bottom) with 329 and 165 stable voxels respectively. The sign and strength of modulation is color-coded by a warm colormap for increased connectivity and cold colormap for decreased connectivity. The zoomed-in image shows that the majority of discriminative voxels in both comparisons were located in the posterior cingulate cortex (PCC) and precuneus. The numbers refer to axial plane zcoordinates in MNI space.

\section{Discussion}

There is considerable interest in both the neurosciences and clinical scenes to understand changes in functional connectivity driven by $10 \mathrm{~Hz}$ rTMS. Here we have used a predictive SVM model approach to identify the time intervals after stimulation that exhibit the most substantial whole-brain functional changes in large-scale network nodes and boundaries. To overcome the issue of high-dimensional data, that is, the number of voxels in node and boundary maps is much greater than the number of subjects in the study, we have applied feature selection using multiple threshold ICA-based masks created from separate fMRI data. Confirming our hypothesis, we have identified connectivity changes related to $10 \mathrm{~Hz}$ rTMS in both network node and boundary maps. Of note, as indicated by cross-validated SVM, changes majorly comprise the PCC, angular gyrus, insula and fusiform gyrus. Accuracy confidence intervals in the classification of boundaries are similarly as substantial as those occurring in nodes. A complex pattern of changes was observed in boundaries alternating between decreases and increases in functional connectivity, which was particularly evident but not limited to, the PCC and precuneus (Figure 7). Such a pattern is reminiscent of the neuronal population dynamics in the cat visual cortex after $10 \mathrm{~Hz}$ rTMS (Kozyrev et al. 2014). The stimulation induced initial suppression of on-going cortical activity, followed by an increase in cortical excitability (long-term potentiation) that lasted about 2-3 hours, but was prone to slow activity fluctuations.

This work extends to the concept of modularity and integration frequently addressed in the field of connectomics (van den Heuvel and Sporns, 2019), both of which focus on the perspective of nodes in the formation and reorganization of networks in cognitive functions 
and in clinical disorders. Most connectomics studies to date have been focused on the strongest points of functional connectivity, "hubs", and the way that such hubs are organized to efficiently propagate information across regions (Crossley et al., 2013; Heuvel and Sporns, 2013). Transition gradients have recently received more attention in the literature (Hirose et al., 2012; Huntenburg et al., 2018; Jbabdi et al., 2013). To the best of our knowledge, the current study is the first to show which changes occur in functional connectivity boundaries and nodes after $10 \mathrm{~Hz}$ rTMS. Indeed, based on their accuracy and confidence intervals, we see that rTMS effects related to boundaries are similarly as substantial as those seen in nodes. The exact function of boundaries is not yet well understood, which might have discouraged its systematic evaluation. It may be speculated that boundaries may act to segregate information within functional regions, or that they may support network stability (Radicchi and Arenas, 2013). Another possible role of boundaries may be supporting functional adaptation of a given region during plastic changes in the mature primate brain. Evidence for this has been shown in primary motor and sensory regions (for review see Florence et al., 1997). In support of this theory are several studies that have shown that rTMS-induced changes in neuronal inhibition can prime cortical networks for the expression of subsequent experience-dependent plasticity (Holtmaat and Svoboda, 2009; Kozyrev et al., 2018; Lenz et al., 2016). The high frequency rTMS potentially creates a cortical state with enhanced plasticity, opening a time window for targeted re-learning of connectivity patterns (Kozyrev et al. 2018).

Causal effects of 1 and $5 \mathrm{~Hz}$ rTMS on global functional connectivity have been explored recently using fMRI (Castrillon et al., 2020; Davis et al., 2017). These studies have found that distant effects, that is, effects relatively far from DLPFC, are determined by connectivity profiles of the stimulation target with macroscopic networks. Excitatory $10 \mathrm{~Hz}$ rTMS in the left DLPFC, as we used in the current study, resulted in multivariate patterns of increases and decreases in functional connectivity. These fluctuations occurred primarily in the PCC, angular gyrus, fusiform gyrus, and insula regions. Each of these regions have been previously shown to be functionally related to the DLPFC (Bokde et al., 2006; Chang and Glover, 2009; Jiang et al., 2015; Taren et al., 2017), reinforcing the notion of distant effects of this stimulation protocol. In the current study, the SVM most substantially identified functional connectivity changes in nodes that occur about $30 \mathrm{~min}$ after stimulation. In contrast, effects related to boundaries were temporally extended up to $45 \mathrm{~min}$. Previous studies that investigated the effects of $10 \mathrm{~Hz}$ rTMS with rsfMRI constrained their analyses to particular seeds or networks 
of interest. Another important advancement provided by the current study is an assessment that started from a global evaluation of $10 \mathrm{~Hz}$ rTMS effects, considering maps of nodes and boundaries at the subject level at different intervals. Cytoarchitectural divisions of the PCC into dorsal and ventral parts have been previously shown to exhibit distinct functional connectivity patterns (Vogt et al., 2006). According to findings from a recent study (Salehi et al., 2020), both PCC and precuneus contain predominantly transient nodes - an entropy class that the authors used to classify changes in network assignments across subjects and brain states. Our findings are consistent with this notion, highlighting that further functional heterogeneity is largely elicited in these regions, particularly in boundaries, after $10 \mathrm{~Hz}$ rTMS.

The importance of individualized characterization of functional brain networks has been highlighted in the literature (Gordon et al., 2017; Laumann et al., 2015; Tavor et al., 2016). These developments may inform future clinical applications based in "precision" or "systems" medicine. Toward this goal, methods applied in the current study might have been advantageous, considering that individual subject analysis of node and boundary maps closely correspond to the original study (Wig et al., 2014). In our study though functional boundaries were not only extracted for the cortical surface. To match the resolution and dimensionality of the node maps, we calculated boundaries on the whole brain. Furthermore, our study is unique in that we used pairwise comparisons of each individualized map type, that is, nodes and boundaries, in the context of a double-blind design controlling for placebo effects. This may have allowed us to identify the contribution of boundaries to the functional changes caused by $10 \mathrm{~Hz}$ rTMS. This individualized approach was followed by SVM, which might have contributed to the identification of the most important effects related to the time intervals and areas.

SVM is a machine learning approach with clear advantages over univariate models. With that said, important preconditions had to be fulfilled to avoid potential biases. One of the main concerns with SVM is to prevent overfitting. The full-resolution rsfMRI was first resampled to 19,973 voxels to reduce the computation time of the spatial correlation matrix - the most computationally demanding step in the algorithm. Splitting the rsfMRI signal into two complementary maps also had the practical benefit of enabling independent assessment using SVM. This also reduced the number of input variables to the model, and thus further prevented overfitting. Secondly, the large number of voxels in both individualized node and 
boundary maps required additional feature selection. While there are several masking algorithms that can locate functional nodes of the brain, to our knowledge, there is no complementary boundary parcellation method associated with any of them. For this reason, we developed a novel approach based on group ICA to reduce the number of voxels included in machine learning. We considered this in our relatively small sample by systematically reducing dimensionality with different levels of feature selection. That is, separate masks controlled by the threshold were created for both nodes and boundaries, which served for feature selection of individual subjects' node and boundary maps. Nevertheless, these masks included common voxels in several regions, including ventral PCC, the boundary area between angular gyrus and fusiform gyrus, and the boundary area between fusiform gyrus and associative visual cortex. These regions are consistent with findings from a recent study that reported on low stability of connectivity regions (Salehi et al., 2020). Some of these regions have been associated with myelin content, particularly the ones that myelinate latest during development (Becker, 1953; Glasser and Essen, 2011). As some of the implicated regions overlap with those with late myelination, this suggests a role for flexible connectivity in learning (Davis et al., 2017).

Our novel feature selection method applied to high dimensional and potentially noisy data is closely related to the recursive feature elimination technique that has been previously applied in neuroimaging (Lu et al., 2016; Wottschel et al., 2019). In our method, voxels/features are not removed based on their rank according to SVM, but rather based on the information obtained from the independent dataset (not included in SVM modeling). Therefore, our approach is less prone to overfitting and inflating performance accuracy. On the other hand, a drawback of our approach is that some fraction of the voxels not included might be potentially informative for classification. Considering our small sample size, the performance of the classifier was low - similar to chance - when all the features were used. For most of the comparisons, there was a substantial drop in the accuracy when more than 1000 voxels were used in the model. This observation may be explained by the presence of too many insignificant voxels and a small sample size. After the optimal number of voxels was reached from unbiased feature selection, further removal of voxels resulted in a drop of the accuracy. This is consistent with previous studies that have used similar machine learning approaches

560 (Wottschel et al., 2019, 2015). Moreover, we investigated estimated accuracy curves for different CV methods as the number of CV folds may influence the accuracy of machine 
learning. That is, threshold values that yielded higher accuracy for leave-three-out, in contrast to leave-one-out CV, are more likely to have inflated accuracy as the training set is smaller. In general, the trained model should underperform or at least yield similar accuracy when trained with less data, otherwise the performance of the model is ambiguous. In this is also seen in the lower accuracy of the leave-three-out in contrast to the leave-one-out CV in our data.

Our work has important limitations that should be considered. As mentioned above, with feature selection not all information derived from both node and boundary maps was considered for classification. Therefore, we cannot exclude that additional areas might have been modulated by $10 \mathrm{~Hz}$ rTMS and, due to the feature selection procedure, are not identified in our results. While it is noteworthy that systematic evaluation of thresholds is a common approach in machine learning, we acknowledge that nodes and boundary masks behave differently when changing threshold values. This may have contributed to the exclusion of regions as part of boundaries at a faster rate than nodes. One possible explanation for this is non-uniform distance between networks which may cause proximal areas in boundaries to interact/overlap at lower thresholds compared to more distant regions in nodes. In addition, an larger sample size may have enabled further methodological improvements such as: (1) increasing the resolution in the masking procedure; (2) consideration of more voxels from the masks; (3) application of non-linear predictive algorithms; (4) better fine-tuning of classifier hyperparameters; and (5) an independent validation dataset to strengthen the reliability of our results.

Our findings provide evidence that SVM classifiers using ICA-based feature selection can identify different spatial definition, direction, and timing in the pattern of fMRI-based brain connectivity changes in functional nodes and boundaries derived from $10 \mathrm{~Hz} \mathrm{rTMS}$ applied to the left DLPFC. Identified nodes and boundaries are located predominantly in the ventral PCC, precuneus, insula, and fusiform gyrus and appear approximately 30 minutes after the stimulation is performed. By dividing the signal into two complementary parts (nodes and boundaries), we highlight the contribution of boundaries to modulatory effects of high frequency rTMS. These findings provide new insights into the currently unknown role of boundaries in network organization, motivating future, related investigations for the advancement of connectomics. 


\section{Acknowledgements}

594

595

596

597

598

599

600

601

602

603

604

605

606

607

608

609

610

611

612

613

614

615

616

617

618

619

620

621

622

623

624

625

626

627

628

629

630

631

632

633

634

635

This work was supported by the German Federal Ministry of Education and Research (Bundesministerium fuer Bildung und Forschung, BMBF: 01 ZX 1507, “PreNeSt - e:Med”).

\section{Conflicts of interest}

The authors declare no conflicts of interest.

\section{References}

Agosta, F., Pievani, M., Geroldi, C., Copetti, M., Frisoni, G.B., Filippi, M., 2012. Resting state fMRI in Alzheimer's disease: beyond the default mode network. Neurobiol. Aging 33, 1564-1578. https://doi.org/10.1016/j.neurobiolaging.2011.06.007

Becker, R.F., 1953. Essay on the cerebral cortex. By Gerhardt von Bonin. Charles C Thomas, Springfield, III. 1950. 150 pp. Am. J. Phys. Anthropol. 11, 441-444. https://doi.org/10.1002/ajpa.1330110317

Bokde, A.L.W., Lopez-Bayo, P., Meindl, T., Pechler, S., Born, C., Faltraco, F., Teipel, S.J., Möller, H.-J., Hampel, H., 2006. Functional connectivity of the fusiform gyrus during a face-matching task in subjects with mild cognitive impairment. Brain 129, 1113-1124. https://doi.org/10.1093/brain/awl051

Castrillon, G., Sollmann, N., Kurcyus (Bieńkowska), K., Razi, A., Krieg, S., Riedl, V., 2020. The physiological effects of noninvasive brain stimulation fundamentally differ across the human cortex. Sci. Adv. 6, eaay2739. https://doi.org/10.1126/sciadv.aay2739

Chang, C., Glover, G.H., 2009. Effects of model-based physiological noise correction on default mode network anti-correlations and correlations. Neurolmage 47, 1448-1459. https://doi.org/10.1016/j.neuroimage.2009.05.012

Chao-Gan, Y., Yu-Feng, Z., 2010. DPARSF: A MATLAB Toolbox for "Pipeline" Data Analysis of RestingState fMRI. Front. Syst. Neurosci. 4. https://doi.org/10.3389/fnsys.2010.00013

Chaplot, S., Patnaik, L.M., Jagannathan, N.R., 2006. Classification of magnetic resonance brain images using wavelets as input to support vector machine and neural network. Biomed. Signal Process. Control 1, 86-92. https://doi.org/10.1016/j.bspc.2006.05.002

Cocchi, L., Sale, M.V., Lord, A., Zalesky, A., Breakspear, M., Mattingley, J.B., 2015. Dissociable effects of local inhibitory and excitatory theta-burst stimulation on large-scale brain dynamics. J. Neurophysiol. 113, 3375-3385. https://doi.org/10.1152/jn.00850.2014

Cohen, A.L., Fair, D.A., Dosenbach, N.U.F., Miezin, F.M., Dierker, D., Van Essen, D.C., Schlaggar, B.L., Petersen, S.E., 2008. Defining functional areas in individual human brains using resting functional connectivity MRI. Neurolmage 41, 45-57. https://doi.org/10.1016/j.neuroimage.2008.01.066

Crossley, N.A., Mechelli, A., Vértes, P.E., Winton-Brown, T.T., Patel, A.X., Ginestet, C.E., McGuire, P., Bullmore, E.T., 2013. Cognitive relevance of the community structure of the human brain functional coactivation network. Proc. Natl. Acad. Sci. U. S. A. 110, 11583-11588. https://doi.org/10.1073/pnas.1220826110

Davis, S.W., Luber, B., Murphy, D.L.K., Lisanby, S.H., Cabeza, R., 2017. Frequency-specific neuromodulation of local and distant connectivity in aging and episodic memory function. Hum. Brain Mapp. 38, 5987-6004. https://doi.org/10.1002/hbm.23803

Eickhoff, S.B., Yeo, B.T.T., Genon, S., 2018. Imaging-based parcellations of the human brain. Nat. Rev. Neurosci. 19, 672-686. https://doi.org/10.1038/s41583-018-0071-7 
Eldaief, M.C., Halko, M.A., Buckner, R.L., Pascual-Leone, A., 2011. Transcranial magnetic stimulation modulates the brain's intrinsic activity in a frequency-dependent manner. Proc. Natl. Acad. Sci. U. S. A. 108, 21229-21234. https://doi.org/10.1073/pnas.1113103109

Evgeniou, T., Pontil, M., 2001. Support Vector Machines: Theory and Applications. pp. 249-257. https://doi.org/10.1007/3-540-44673-7_12

Florence, S.L., Jain, N., Kaas, J.H., 1997. Plasticity of Somatosensory Cortex in Primates. Semin. Neurosci. 9, 3-12. https://doi.org/10.1006/smns.1997.0101

George, M.S., Stallings, L.E., Speer, A.M., Nahas, Z., Spicer, K.M., Vincent, D.J., Bohning, D.E., Cheng, K.T., Molloy, M., Teneback, C.C., Risch, S.C., 1999. Prefrontal repetitive transcranial magnetic stimulation (rTMS) changes relative perfusion locally and remotely. Hum. Psychopharmacol. Clin. Exp. 14, 161-170. https://doi.org/10.1002/(SICI)1099-1077(199904)14:3<161::AIDHUP73>3.0.CO;2-2

Glasser, M.F., Coalson, T.S., Robinson, E.C., Hacker, C.D., Harwell, J., Yacoub, E., Ugurbil, K., Andersson, J., Beckmann, C.F., Jenkinson, M., Smith, S.M., Van Essen, D.C., 2016. A multimodal parcellation of human cerebral cortex. Nature 536, 171-178. https://doi.org/10.1038/nature18933

Glasser, M.F., Essen, D.C.V., 2011. Mapping Human Cortical Areas In Vivo Based on Myelin Content as Revealed by T1- and T2-Weighted MRI. J. Neurosci. 31, 11597-11616. https://doi.org/10.1523/JNEUROSCI.2180-11.2011

Gollo, L.L., Roberts, J.A., Cocchi, L., 2017. Mapping how local perturbations influence systems-level brain dynamics. Neurolmage 160, 97-112. https://doi.org/10.1016/j.neuroimage.2017.01.057

Gordon, E.M., Laumann, T.O., Adeyemo, B., Huckins, J.F., Kelley, W.M., Petersen, S.E., 2016. Generation and Evaluation of a Cortical Area Parcellation from Resting-State Correlations. Cereb. Cortex N. Y. N 1991 26, 288-303. https://doi.org/10.1093/cercor/bhu239

Gordon, E.M., Laumann, T.O., Gilmore, A.W., Newbold, D.J., Greene, D.J., Berg, J.J., Ortega, M., HoytDrazen, C., Gratton, C., Sun, H., Hampton, J.M., Coalson, R.S., Nguyen, A.L., McDermott, K.B., Shimony, J.S., Snyder, A.Z., Schlaggar, B.L., Petersen, S.E., Nelson, S.M., Dosenbach, N.U.F., 2017. Precision Functional Mapping of Individual Human Brains. Neuron 95, 791-807.e7. https://doi.org/10.1016/j.neuron.2017.07.011

Goya-Maldonado, R., Brodmann, K., Keil, M., Trost, S., Dechent, P., Gruber, O., 2016. Differentiating unipolar and bipolar depression by alterations in large-scale brain networks. Hum. Brain Mapp. 37, 808-818. https://doi.org/10.1002/hbm.23070

Heuvel, M.P. van den, Sporns, O., 2013. Network hubs in the human brain. Trends Cogn. Sci. 17, 683696. https://doi.org/10.1016/j.tics.2013.09.012

Hirose, S., Watanabe, T., Jimura, K., Katsura, M., Kunimatsu, A., Abe, O., Ohtomo, K., Miyashita, Y., Konishi, S., 2012. Local Signal Time-Series during Rest Used for Areal Boundary Mapping in Individual Human Brains. PLOS ONE 7, e36496. https://doi.org/10.1371/journal.pone.0036496

Holtmaat, A., Svoboda, K., 2009. Experience-dependent structural synaptic plasticity in the mammalian brain. Nat. Rev. Neurosci. 10, 647-658. https://doi.org/10.1038/nrn2699

Huntenburg, J.M., Bazin, P.-L., Margulies, D.S., 2018. Large-Scale Gradients in Human Cortical Organization. Trends Cogn. Sci. 22, 21-31. https://doi.org/10.1016/j.tics.2017.11.002

Jbabdi, S., Sotiropoulos, S.N., Behrens, T.E., 2013. The topographic connectome. Curr. Opin. Neurobiol., Macrocircuits 23, 207-215. https://doi.org/10.1016/j.conb.2012.12.004

Jiang, J., Beck, J., Heller, K., Egner, T., 2015. An insula-frontostriatal network mediates flexible cognitive control by adaptively predicting changing control demands. Nat. Commun. 6, 8165. https://doi.org/10.1038/ncomms9165

Khosla, M., Jamison, K., Ngo, G.H., Kuceyeski, A., Sabuncu, M.R., 2019. Machine learning in restingstate $\mathrm{fMRI}$ analysis. Magn. Reson. Imaging 64, 101-121. https://doi.org/10.1016/j.mri.2019.05.031 
Kohoutová, L., Heo, J., Cha, S., Lee, S., Moon, T., Wager, T.D., Woo, C.-W., 2020. Toward a unified framework for interpreting machine-learning models in neuroimaging. Nat. Protoc. 15, 13991435. https://doi.org/10.1038/s41596-019-0289-5

Kolade, O., Olayinka, A.A., Ovie, U., 2014. Fingerprint Database Optimization Using Watershed Transformation Algorithm. Open J. Optim. 3, 59-67. https://doi.org/10.4236/ojop.2014.34006

Kong, R., Li, J., Orban, C., Sabuncu, M.R., Liu, H., Schaefer, A., Sun, N., Zuo, X.-N., Holmes, A.J., Eickhoff, S.B., Yeo, B.T.T., 2019. Spatial Topography of Individual-Specific Cortical Networks Predicts Human Cognition, Personality, and Emotion. Cereb. Cortex N. Y. N 1991 29, 25332551. https://doi.org/10.1093/cercor/bhy123

Kozyrev, V., Eysel, U.T., Jancke, D., 2014. Voltage-sensitive dye imaging of transcranial magnetic stimulation-induced intracortical dynamics. Proc. Natl. Acad. Sci. U. S. A. 111, 13553-13558. https://doi.org/10.1073/pnas.1405508111

Kozyrev, V., Staadt, R., Eysel, U.T., Jancke, D., 2018. TMS-induced neuronal plasticity enables targeted remodeling of visual cortical maps. Proc. Natl. Acad. Sci. U. S. A. 115, 6476-6481. https://doi.org/10.1073/pnas.1802798115

Laumann, T.O., Gordon, E.M., Adeyemo, B., Snyder, A.Z., Joo, S.J., Chen, M.-Y., Gilmore, A.W., McDermott, K.B., Nelson, S.M., Dosenbach, N.U.F., Schlaggar, B.L., Mumford, J.A., Poldrack, R.A., Petersen, S.E., 2015. Functional System and Areal Organization of a Highly Sampled Individual Human Brain. Neuron 87, 657-670. https://doi.org/10.1016/j.neuron.2015.06.037

Lenz, M., Galanis, C., Müller-Dahlhaus, F., Opitz, A., Wierenga, C.J., Szabó, G., Ziemann, U., Deller, T., Funke, K., Vlachos, A., 2016. Repetitive magnetic stimulation induces plasticity of inhibitory synapses. Nat. Commun. 7, 10020. https://doi.org/10.1038/ncomms10020

Liu, P., Choo, K.-K.R., Wang, L., Huang, F., 2017. SVM or deep learning? A comparative study on remote sensing image classification. Soft Comput. 21. https://doi.org/10.1007/s00500-0162247-2

Lu, X., Yang, Y., Wu, F., Gao, M., Xu, Y., Zhang, Y., Yao, Y., Du, X., Li, C., Wu, L., Zhong, X., Zhou, Y., Fan, N., Zheng, Y., Xiong, D., Peng, H., Escudero, J., Huang, B., Li, X., Ning, Y., Wu, K., 2016. Discriminative analysis of schizophrenia using support vector machine and recursive feature elimination on structural MRI images. Medicine (Baltimore) 95, e3973. https://doi.org/10.1097/MD.0000000000003973

Miao, J., Niu, L., 2016. A Survey on Feature Selection. Procedia Comput. Sci. 91, 919-926. https://doi.org/10.1016/j.procs.2016.07.111

Peraza, L.R., Kaiser, M., Firbank, M., Graziadio, S., Bonanni, L., Onofrj, M., Colloby, S.J., Blamire, A., O'Brien, J., Taylor, J.-P., 2014. fMRI resting state networks and their association with cognitive fluctuations in dementia with Lewy bodies. Neurolmage Clin. 4, 558-565. https://doi.org/10.1016/j.nicl.2014.03.013

Power, J.D., Cohen, A.L., Nelson, S.M., Wig, G.S., Barnes, K.A., Church, J.A., Vogel, A.C., Laumann, T.O., Miezin, F.M., Schlaggar, B.L., Petersen, S.E., 2011. Functional network organization of the human brain. Neuron 72, 665-678. https://doi.org/10.1016/j.neuron.2011.09.006

Power, J.D., Plitt, M., Laumann, T.O., Martin, A., 2017. Sources and implications of whole-brain fMRI signals in humans. Neurolmage 146, 609-625. https://doi.org/10.1016/j.neuroimage.2016.09.038

Radicchi, F., Arenas, A., 2013. Abrupt transition in the structural formation of interconnected networks. Nat. Phys. 9, 717-720. https://doi.org/10.1038/nphys2761

Sale, M.V., Mattingley, J.B., Zalesky, A., Cocchi, L., 2015. Imaging human brain networks to improve the clinical efficacy of non-invasive brain stimulation. Neurosci. Biobehav. Rev. 57, 187-198. https://doi.org/10.1016/j.neubiorev.2015.09.010

Salehi, M., Karbasi, A., Barron, D.S., Scheinost, D., Constable, R.T., 2020. Individualized functional networks reconfigure with cognitive state. Neurolmage 206, 116233. https://doi.org/10.1016/j.neuroimage.2019.116233 
Satterthwaite, T.D., Elliott, M.A., Gerraty, R.T., Ruparel, K., Loughead, J., Calkins, M.E., Eickhoff, S.B., Hakonarson, H., Gur, R.C., Gur, R.E., Wolf, D.H., 2013. An improved framework for confound regression and filtering for control of motion artifact in the preprocessing of resting-state functional connectivity data. Neurolmage 64, 240-256. https://doi.org/10.1016/j.neuroimage.2012.08.052

Saxe, R., Moran, J.M., Scholz, J., Gabrieli, J., 2006. Overlapping and non-overlapping brain regions for theory of mind and self reflection in individual subjects. Soc. Cogn. Affect. Neurosci. 1, 229234. https://doi.org/10.1093/scan/nsl034

Singh, A., Erwin-Grabner, T., Sutcliffe, G., Antal, A., Paulus, W., Goya-Maldonado, R., 2019. Personalized repetitive transcranial magnetic stimulation temporarily alters default mode network in healthy subjects. Sci. Rep. 9, 5631. https://doi.org/10.1038/s41598-019-42067-3

Singh, A., Erwin-Grabner, T., Sutcliffe, G., Paulus, W., Dechent, P., Antal, A., Goya-Maldonado, R., 2020. Default mode network alterations after intermittent theta burst stimulation in healthy subjects. Transl. Psychiatry 10, 75. https://doi.org/10.1038/s41398-020-0754-5

Smith, S.M., Vidaurre, D., Beckmann, C.F., Glasser, M.F., Jenkinson, M., Miller, K.L., Nichols, T.E., Robinson, E.C., Salimi-Khorshidi, G., Woolrich, M.W., Barch, D.M., Uğurbil, K., Van Essen, D.C., 2013. Functional connectomics from resting-state fMRI. Trends Cogn. Sci. 17, 666-682. https://doi.org/10.1016/j.tics.2013.09.016

Stanley, M.L., Moussa, M.N., Paolini, B., Lyday, R.G., Burdette, J.H., Laurienti, P.J., 2013. Defining nodes in complex brain networks. Front. Comput. Neurosci. 7. https://doi.org/10.3389/fncom.2013.00169

Taren, A.A., Gianaros, P.J., Greco, C.M., Lindsay, E.K., Fairgrieve, A., Brown, K.W., Rosen, R.K., Ferris, J.L., Julson, E., Marsland, A.L., Creswell, J.D., 2017. Mindfulness Meditation Training and Executive Control Network Resting State Functional Connectivity: A Randomized Controlled Trial. Psychosom. Med. 79, 674-683. https://doi.org/10.1097/PSY.0000000000000466

Tavor, I., Parker Jones, O., Mars, R.B., Smith, S.M., Behrens, T.E., Jbabdi, S., 2016. Task-free MRI predicts individual differences in brain activity during task performance. Science 352, 216220. https://doi.org/10.1126/science.aad8127

Togo, H., Rokicki, J., Yoshinaga, K., Hisatsune, T., Matsuda, H., Haga, N., Hanakawa, T., 2017. Effects of Field-Map Distortion Correction on Resting State Functional Connectivity MRI. Front. Neurosci. 11. https://doi.org/10.3389/fnins.2017.00656

Tourville, J., Nieto-Castañón, A., Heyne, M., Guenther, F., 2019. Functional Parcellation of the Speech Production Cortex. J. Speech Lang. Hear. Res. JSLHR. https://doi.org/10.1044/2019_JSLHR-SCSMC7-18-0442

Valchev, N., Ćurčić-Blake, B., Renken, R.J., Avenanti, A., Keysers, C., Gazzola, V., Maurits, N.M., 2015. cTBS delivered to the left somatosensory cortex changes its functional connectivity during rest. Neurolmage 114, 386-397. https://doi.org/10.1016/j.neuroimage.2015.04.017

van den Heuvel, M.P., Sporns, O., 2019. A cross-disorder connectome landscape of brain dysconnectivity. Nat. Rev. Neurosci. 20, 435-446. https://doi.org/10.1038/s41583-019-01776

Van Dijk, K.R.A., Sabuncu, M.R., Buckner, R.L., 2012. The influence of head motion on intrinsic functional connectivity MRI. Neurolmage 59, 431-438. https://doi.org/10.1016/j.neuroimage.2011.07.044

Vogt, B.A., Vogt, L., Laureys, S., 2006. Cytology and functionally correlated circuits of human posterior cingulate areas. Neurolmage 29, 452-466. https://doi.org/10.1016/j.neuroimage.2005.07.048

Watanabe, T., Hanajima, R., Shirota, Y., Ohminami, S., Tsutsumi, R., Terao, Y., Ugawa, Y., Hirose, S., Miyashita, Y., Konishi, S., Kunimatsu, A., Ohtomo, K., 2014. Bidirectional effects on interhemispheric resting-state functional connectivity induced by excitatory and inhibitory repetitive transcranial magnetic stimulation. Hum. Brain Mapp. 35, 1896-1905. https://doi.org/10.1002/hbm.22300 
Wei, Y., Chang, M., Womer, F.Y., Zhou, Q., Yin, Z., Wei, S., Zhou, Y., Jiang, X., Yao, X., Duan, J., Xu, K., Zuo, X.-N., Tang, Y., Wang, F., 2018. Local functional connectivity alterations in schizophrenia, bipolar disorder, and major depressive disorder. J. Affect. Disord. 236, 266-273. https://doi.org/10.1016/j.jad.2018.04.069

Wig, G.S., Laumann, T.O., Cohen, A.L., Power, J.D., Nelson, S.M., Glasser, M.F., Miezin, F.M., Snyder, A.Z., Schlaggar, B.L., Petersen, S.E., 2014. Parcellating an individual subject's cortical and subcortical brain structures using snowball sampling of resting-state correlations. Cereb. Cortex N. Y. N 1991 24, 2036-2054. https://doi.org/10.1093/cercor/bht056

Wottschel, V., Alexander, D.C., Kwok, P.P., Chard, D.T., Stromillo, M.L., De Stefano, N., Thompson, A.J., Miller, D.H., Ciccarelli, O., 2015. Predicting outcome in clinically isolated syndrome using machine learning. Neurolmage Clin. 7, 281-287. https://doi.org/10.1016/j.nicl.2014.11.021

Wottschel, V., Chard, D.T., Enzinger, C., Filippi, M., Frederiksen, J.L., Gasperini, C., Giorgio, A., Rocca, M.A., Rovira, A., De Stefano, N., Tintoré, M., Alexander, D.C., Barkhof, F., Ciccarelli, O., MAGNIMS study group and the EuroPOND consortium, 2019. SVM recursive feature elimination analyses of structural brain MRI predicts near-term relapses in patients with clinically isolated syndromes suggestive of multiple sclerosis. Neurolmage Clin. 24, 102011. https://doi.org/10.1016/j.nicl.2019.102011

Yan, C.-G., Cheung, B., Kelly, C., Colcombe, S., Craddock, R.C., Di Martino, A., Li, Q., Zuo, X.-N., Castellanos, F.X., Milham, M.P., 2013. A Comprehensive Assessment of Regional Variation in the Impact of Head Micromovements on Functional Connectomics. Neurolmage 76, 183201. https://doi.org/10.1016/j.neuroimage.2013.03.004

Yeo, B.T.T., Krienen, F.M., Sepulcre, J., Sabuncu, M.R., Lashkari, D., Hollinshead, M., Roffman, J.L., Smoller, J.W., Zöllei, L., Polimeni, J.R., Fischl, B., Liu, H., Buckner, R.L., 2011. The organization of the human cerebral cortex estimated by intrinsic functional connectivity. J. Neurophysiol. 106, 1125-1165. https://doi.org/10.1152/jn.00338.2011 

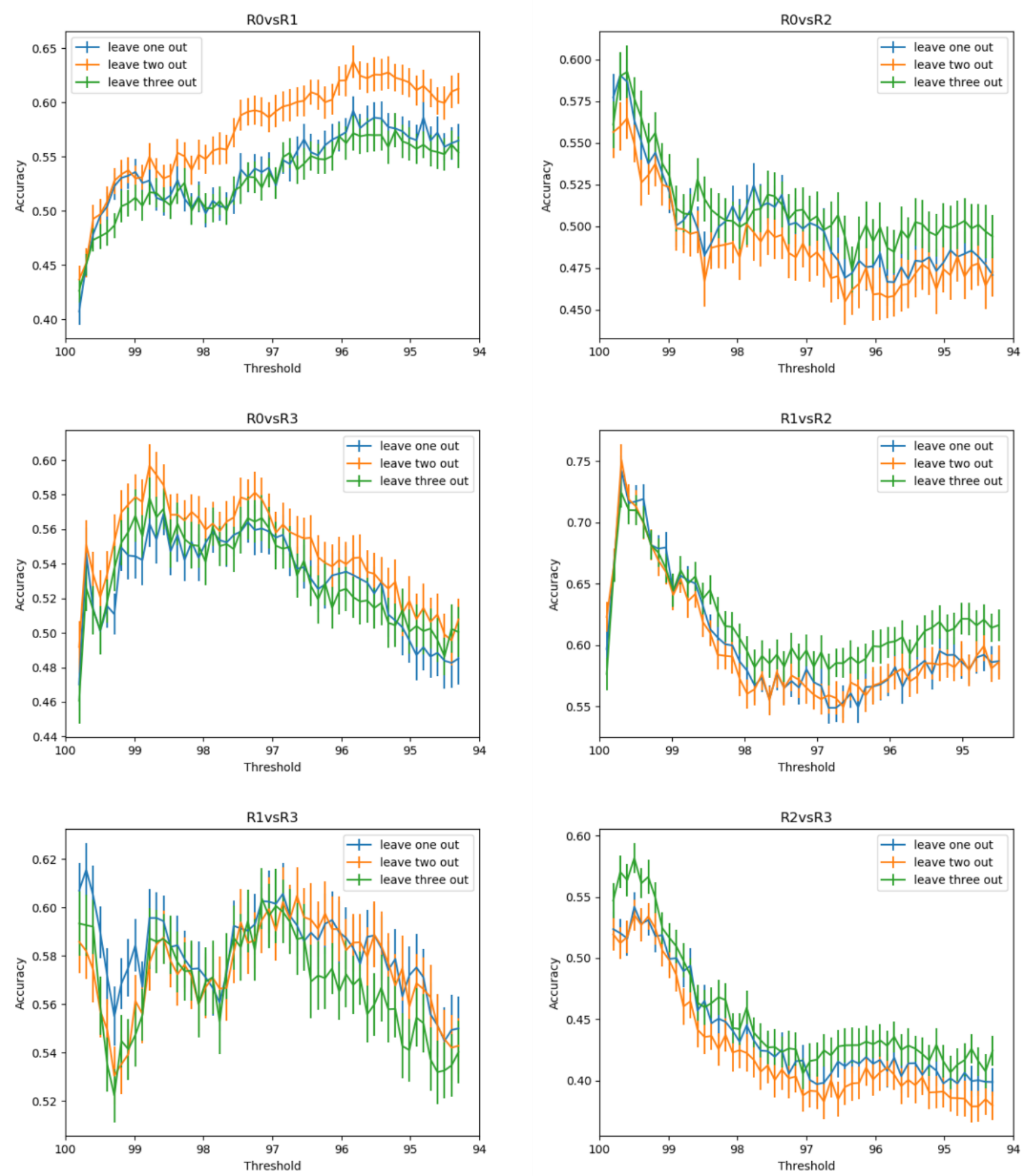

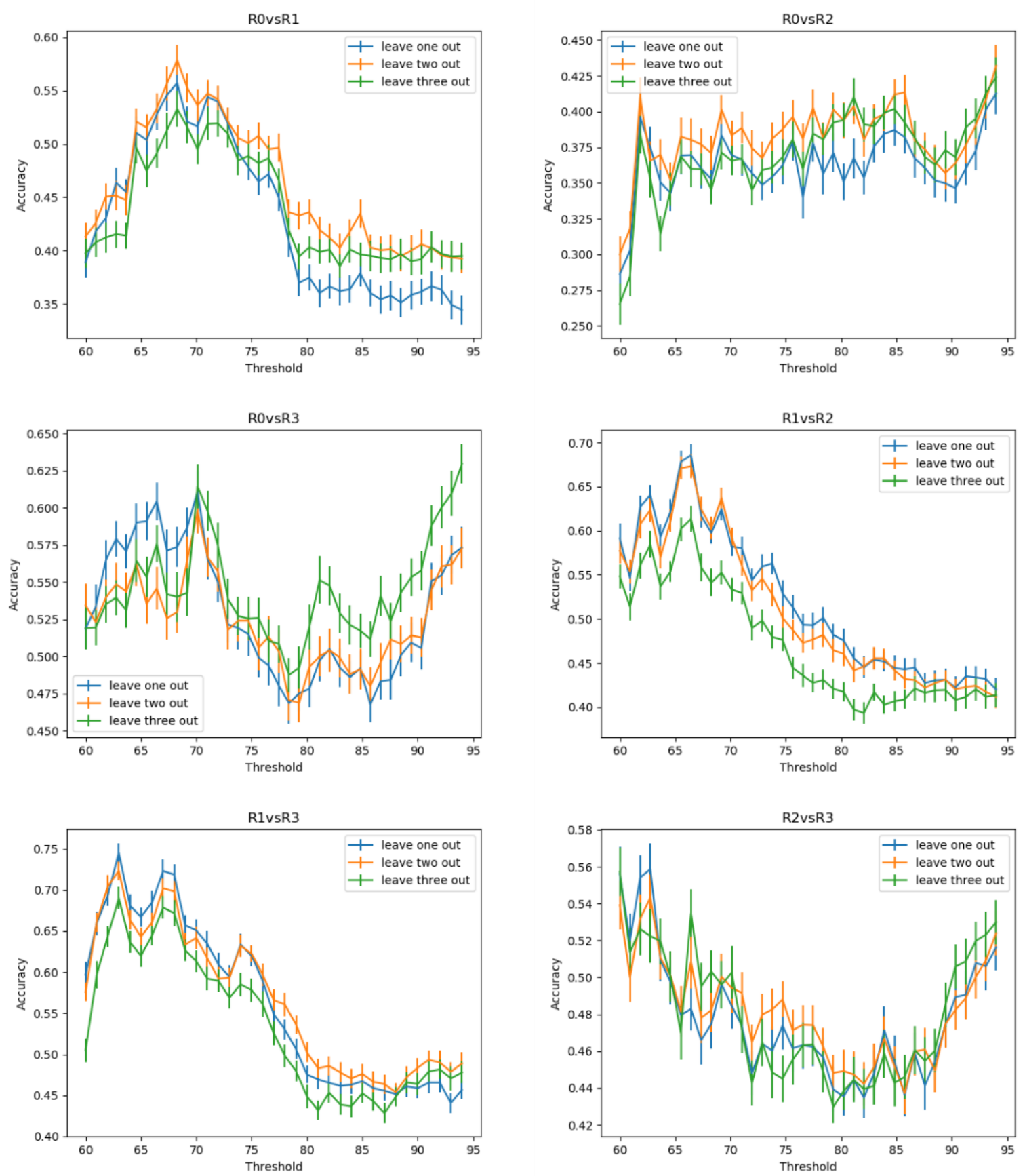

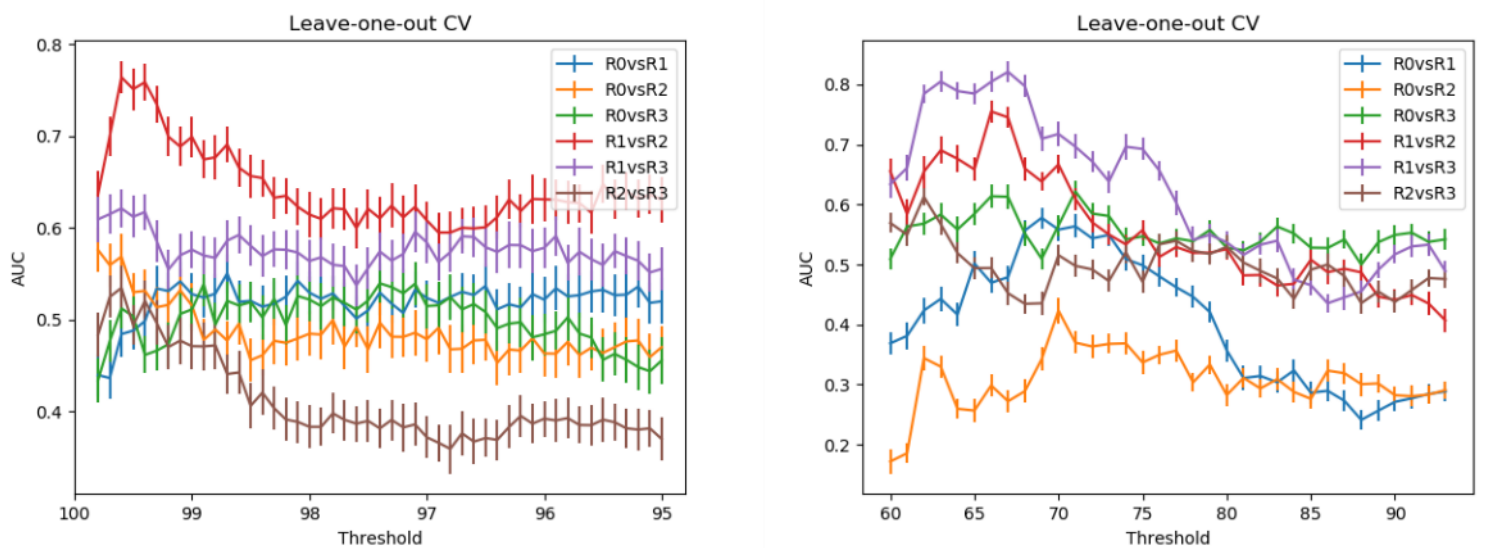

835 Supplementary Figure 3: Area under the curve (AUC) of all comparisons for node (left) and 
Supplementary Table 1: Results for the top comparisons under leave-one-out CV for both nodes and boundaries

\begin{tabular}{|l|l|l|l|l|}
\hline Column1 & $\begin{array}{l}\text { Top } \\
\text { comparison }\end{array}$ & $\begin{array}{l}\text { Number } \\
\text { of voxels }\end{array}$ & $\begin{array}{l}\text { SVM accuracy }(95 \% \text { CI) } \\
{[\%]}\end{array}$ & $\begin{array}{l}\text { SVM AUC (95\% CI) } \\
{[\%]}\end{array}$ \\
\hline $\begin{array}{l}\text { Snowballing peak } \\
\text { density }\end{array}$ & R1 vs. R2 & 764 & $74.2(73.0-75.4)$ & $76.4(74.6-78.1)$ \\
\hline \multirow{2}{*}{ Boundary mapping } & R1 vs. R2 & 346 & $68.5(67.2-69.8)$ & $74.5(72.7-86.3)$ \\
\cline { 2 - 6 } & R1 vs. R3 & 174 & $74.5(73.4-75.6)$ & $80.5(78.7-82.2)$ \\
\hline
\end{tabular}

851

853 Supplementary Table 2: Accuracies of the SVM trained on head mean frame-to-frame displacement for real and sham rTMS

\begin{tabular}{|l|l|l|l|l|l|l|}
\hline \multicolumn{3}{|c|}{ Accuracy Real } & \multicolumn{3}{c}{ Accuracy Sham } & Accuracy Real - Sham \\
\hline Condition & Mean & Std & Mean & Std & Mean & Std \\
\hline R0 vs. R1 & 0.50 & 0.15 & 0.50 & 0.29 & 0.57 & 0.27 \\
\hline R0 vs. R2 & 0.52 & 0.10 & 0,54 & 0.20 & 0.52 & 0.31 \\
\hline R0 vs. R3 & 0.52 & 0.10 & 0.50 & 0.00 & 0.35 & 0.23 \\
\hline R1 vs. R2 & 0.54 & 0.14 & 0.54 & 0.20 & 0.46 & 0.20 \\
\hline R1 vs. R3 & 0.57 & 0.17 & 0.54 & 0.14 & 0.52 & 0.23 \\
\hline R2 vs. R3 & 0.50 & 0.00 & 0.50 & 0.00 & 0.50 & 0.00 \\
\hline
\end{tabular}

